Aysegul Baysak ${ }^{1}$

Deniz Akpinar ${ }^{2}$

Kenan Can Ceylan ${ }^{3}$

Gulfem Ece ${ }^{4}$

Elif Duman ${ }^{5}$

Adnan Tolga $\mathrm{Oz}^{6}$

Cem Ece ${ }^{7}$

\section{Tuberculosis cases presenting with spontaneous hemopneumothorax and hypotension}

\author{
${ }^{1}$ Medipol University School of Medicine, Camlica Hospital, Department of Chest Diseases, Istanbul/Turkey \\ ${ }^{2}$ Tekirdag Public Hospital, Chest Surgery Clinic, Tekirdag, Turkey \\ 3Dr. Suat Seren Chest Diseases and Thoracic Surgery Research and Training Hospital, Department of Thoracic Surgery, \\ Izmir, Turkey \\ ${ }^{4}$ Izmir University of Economics, School of Medicine Medicalpark Izmir Hospital, Department of Medical Microbiology, \\ Izmir, Turkey \\ ${ }^{5}$ Samsun Teaching and Research Hospital 2nd Thoracic Surgery Clinic, Samsun, Turkey \\ ${ }^{6}$ Medicalpark Izmir Hospital, Department of Chest Diseases,Izmir,Turkey \\ ${ }^{7}$ Izmir Tinaztepe University, Tinaztepe Galen Hospital, Department of Anesthesiology and Reanimation,Izmir, Turkey
}

Article history

Received: 8 June 2020; Revision Requested: 21 September 2020; Revision Received: 21 October 2020; Accepted: 5 November

2020; Published: 2 January 2021

\section{Sir,}

Tuberculosis (TB) is the second most common fatal infectious disease, following human immunodeficiency virus/ acquired immunodeficiency syndrome (HIV/AIDS), in the adult population. Socio-economic deprivation, immigration, wars, omission of tuberculosis control programs, and HIV/AIDS epidemics have led to an increase in the incidence of tuberculosis. The World Health Organization (WHO) publishes a global tuberculosis report annually. The 2013 report stated that, in 2012, 8.6 million people developed TB, and 1.3 million people died from the disease, including 320,000 deaths among HIV-positive individuals [1].

As a rare complication of spontaneous pneumothorax, spontaneous hemopneumothorax (SHP) is an emergency condition that can be life threatening because of active bleeding in the pleural space; the condition has a high mortality rate and requires early diagnosis and treatment $[2,3]$. A report has suggested that TB will not cause SHP due to thick and extensive pleural adhesions [4].

We present here four cases with SHP secondary to a hypovolemic shock condition due to hemorrhage, together with a literature review due to the rarity of this condition and discuss applicable treatment approaches. Pleural biopsy specimens were stained with Kinyoun and cultivated on Löwenstein-Jensen medium. Cultivation was also performed using a liquid automatized Bactec 460 TB system (Becton-Dickinson).

The first patient was a 34-year-old male admitted to the Emergency Room (ER) because of pain in his right chest and back. With no history of trauma, the patient's physical examination showed diminished breath sounds in the right hemith-

Correspondence:

Aysegul Baysak

Medipol University School of Medicine, Camlica Hospital, Department of Chest Diseases,

Istanbul/Turkey

E-mail:drbaysak@gmail.com orax. His chest $\mathrm{X}$-ray revealed an appearance consistent with hydrothorax with a partial pneumothorax line on the right (Figure 1A). Laboratory findings reported the hemoglobin level of $10.4 \mathrm{~g} / \mathrm{dL}$ and a hematocrit level of $31.4 \%$. No pathological data was detected in coagulation parameters. Tube thoracostomy (TT) was performed on the right after a preliminary diagnosis of SHP and obtained 1000 cc of fresh blood. Emergency right thoracotomy was performed following $300 \mathrm{ml}$ of hemorrhage during the first hour, a clinical condition associated with hypotensive and hypovolemic shock $(80 / 50 \mathrm{mmHg})$; a hemoglobin value reduced to $8.5 \mathrm{~g} / \mathrm{dL}$ and a hematocrit value to $25.2 \%$ in the clinical follow up. Exploration showed that the apex of the upper lobe was adherent to the chest wall with a small adhesion, and aberrant vessels with diameters of $\sim 0.4 \mathrm{~mm}$ at this site and an actively bleeding varicose pattern between the chest wall and pleura were identified. A suture procedure was carried out, and three units of blood were transfused. TB treatment was initiated two months after the diagnosis by histopathological and microbiological examinations of pleural biopsy specimens during emergent thoracotomy procedure.

The second patient was a 25-year-old male admitted to the ER with complaints of shortness of breath and sudden pain in the right chest showed diminished breath sounds on the right in chest auscultation. His chest X-ray showed a partial pneumothorax line on the right consistent with hydrothorax (Figure 1B). The patient had no history of trauma. Laboratory findings reported a hemoglobin level of $12.6 \mathrm{~g} / \mathrm{dL}$, and a hematocrit level of $36.8 \%$. Tा was performed on the right after a preliminary diagnosis of SHP. Air drainage was initially performed and yielded 700 cc of hemorrhagic fluid. Emergency right thoracotomy was performed following $300 \mathrm{ml}$ of hemorrhage during the first hour followed by $200 \mathrm{ml}$ of hemorrhage per hour, a clinical condition associated with hypovolemic hypotension $(90 / 50 \mathrm{mmHg})$ with a hemoglobin value of $9.4 \mathrm{~g} /$ $\mathrm{dL}$ and a hematocrit value of $28.2 \%$ in his clinical follow up. Exploration found a cavity lesion with a dimension of $4 \times 3 \times 2$ 

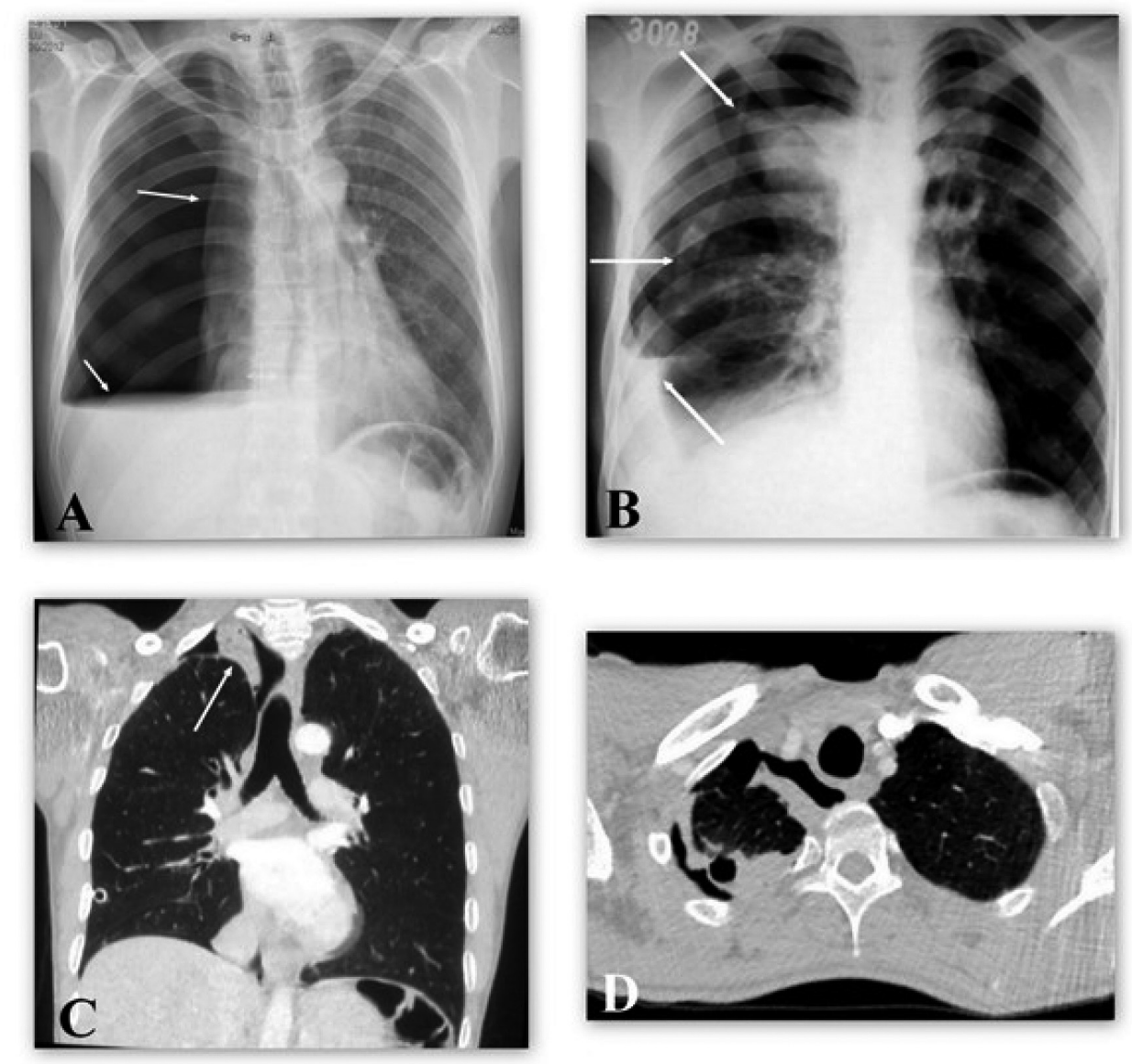

\begin{tabular}{l|l} 
Figure 1 & The chest $\mathrm{X}$-ray and $\mathrm{CT}$ images of the patients
\end{tabular}

$\mathrm{cm}$, ruptured to the pleura in the apical segment of the upper lobe, and segmentectomy was performed. Clinical sample revealed acid fast bacilli (AFB). TB treatment was initiated due to this result by the Chest Diseases Department (CDD) and microbiological evaluation reported tuberculosis after two months.

The third patient was a 28-year-old male admitted to the ER with complaints of pain in the left chest and shortness of breath. With no history of trauma, the patient's thoracic radiograph showed an increased density varying with partial pneumothorax on the left. The bleeding profile and biochemical values were within normal limits and a hemoglobin level of $11.5 \mathrm{~g} / \mathrm{dL}$, and a hematocrit level of $32.4 \%$. $\Pi$ was performed on the left hemithorax. Air drainage was initially performed, yielding 1250 cc of hemorrhagic fluid. Emergency video-assisted thoracoscopic surgery (VATS) was performed in the left hemithorax following $250 \mathrm{ml}$ of hemorrhagic fluid drainage during the first hour due to hypotensive and hypovolemic condition $(90 / 40 \mathrm{mmHg})$, and a hemoglobin value reduced to $9.1 \mathrm{~g} / \mathrm{dL}$ and a hematocrit to 27.2\%. Exploration found bullous lesions, with a maximum diameter $2 \times 2 \mathrm{~cm}$, in the apical segment of the upper lobe and an actively bleeding adhesion lesion in the apical region of the hemithorax. The bullous lesions were excised, and bridging ligation was performed. The patient was followed up by the CDD upon detection of AFB in his sputum and treatment was initiated thereafter.

The fourth patient was a 43-year-old male with no history of trauma who was admitted to the ER because of breath difficulty and sudden pain in the right chest. He was hypotensive $(90 / 50 \mathrm{mmHg})$, dyspneic, and tachypneic, with no breath sounds heard in the right hemithorax. His chest radiography was consistent with total pneumothorax in the right and hydrothorax and deletion of the diaphragm contours. The bleeding profile and biochemical values were within normal limits, a hemoglobin level of $9.1 \mathrm{~g} / \mathrm{dL}$, a hematocrit level of $29.4 \%$, and a platelet count of $435,000 / \mathrm{mm}^{3}$. $\Pi$ was performed on the right hemithorax. Intravenous fluid replacement was initiated. Air drainage was initially performed, yielding 1200 cc of fresh blood. Air discharge and hemorrhagic fluid drainage were stopped at the first hour, and no additional surgical interventions were considered due to his hypotensive condition and improved dyspnea. Two units of blood were transfused upon the decrease of the hemoglobin value to $8.3 \mathrm{~g} / \mathrm{dL}$ and hemat- 
ocrit to $26.4 \%$. The patient was followed up by the CDD upon detection of AFB in his sputum and a high level of adenosine deaminase (ADA: $120 \mathrm{U} / \mathrm{L}$ ) in the pleural fluid; TB treatment was initiated by the CDD (Figure $1 \mathrm{C}$ and $1 \mathrm{D}$ ).

Although hemopneumothorax is typically a result of trauma, SHP is not associated with a trauma history. Associated with pneumothorax, SHP involves $\geq 400-\mathrm{ml}$ bleeding in the pleural cavity [4]. SHP is observed mostly in the adolescent age group and may cause life-threatening bleeding [2]. The incidence of SHP varies between 0.5\% and 12\% [5]. Our clinic treated 184 spontaneous pneumothorax patients between 2008 and 2013, only six of whom (3.2\%) were diagnosed with SHP. SHP secondary to TB was found in four patients.

Three bleeding mechanisms were defined in SHP: first, the lysis of adhesions between visceral and parietal pleura; second, the rupture of congenital aberrant vessels between the parietal pleura and bulla; third, the rupture of the vascularized bullae. Some patients were found to have abnormal vascularization between the lung and parietal pleura [3]. TB, like other chronic infections, can cause abnormal vascularization connecting the parietal pleura and chest wall, leading to aneurysms and intrathoracic hemorrhage if the vessels rupture. This appears to be the explanation for the massive bleeding in two of the cases (Cases 1 and 3). Soo-Kim et al. reported their 12-year experience with SHP [4]. They showed that pleural adhesions after TB do not cause SHP because they are thick and extensive. In two of our cases, very little thickening was observed in the pleura, which could be easily decorticated, whereas SHP was caused by cavity perforation in one of our patients.

SHP cannot compensate for even minimal bleeding because the lung is collapsed, which can lead to severe blood loss. Tube thoracostomy should be the first choice in the treatment of SHP. Studies have demonstrated that only tube thoracostomy with conservative treatment is enough for the treatment of SHP in some patients [3]. Fluid and blood replacement, where necessary, should be started aimed at the stabilization of hemodynamics, the patient should be closely followed, and the need for emergency surgical intervention should be considered [6]. Persistent hemothorax $\geq 200 \mathrm{~mL} / \mathrm{h}$ for consecutive 3 hours, persistent air leak, impaired lung expansion, and empyema are indications for thoracotomy or VATS [7].

\section{FUNDING}

None to declare.

\section{CONFLICTS OF INTEREST}

The authors declare that they have no conflict of interest
2. Hsu CC, Shih CS, Hsu CP, Chen PR. Spontaneous hemopneumothorax revisited: Clinical approach and systemic review of the literature. Ann Thorac Surg. 2005;80:1859-63 doi:10.1016/j. athoracsur.2005.04.052

3. Kakaris S, Athanassiadi K, Vassilikos K, Skottis I.Spontaneous hemopneumothorax: a rare but life-threatening entity. Eur J Cardiothorac Surg. 2004;25(5):856-8 doi:10.1016/j.ejcts.2004.02.002

4. Kim ES, Kang JY, Pyo CH, Jeon EY, Lee WB. 12-year experience of spontaneous hemopneumothorax. Ann Thorac Cardiovasc Surg. 2008;14(3):149-53. PMID:18577892

5. Basoglu A, Celik B, Yetim TD. Massive spontaneous hemopneumothorax complicating rheumatoid lung disease. Ann Thorac Surg. 2007;83(4):1521-3 doi:10.1016/j.athoracsur.2006.09.052

6. Çobanoğlu U, Sayır F, Sertoğullarından B, Mergan D, Aydın İ. Spontan hemopnömotoraks: 8 olgunun analizi. J Clin Anal Med 2012;3(1):59-2 doi:10.4328/JCAM.561

7. Hwong TM, Ng CS, Lee TW, Wan S, Sihoe AD, Wan IY, Arifi AA, Yim AP. Video-assisted thoracic surgery for primary spontaneous hemopneumothorax. Eur J Cardiothorac Surg 2004;26:893- 6 doi:10.1016/j.ejcts.2004.05.014

\section{REFERENCES}

1. Global Tuberculosis Report 2016. http://www.who.int/tb/publications/global_report/en accession date:13.06.2017 